\title{
Fall-Winter Current Reversals on the Texas-Louisiana Continental Shelf
}

\author{
Peter P. CHU \\ Naval Ocean-Atmospheric Prediction Laboratory, Department of Oceanography, Naval Postgraduate School, Monterey, California \\ LEONID M. IvANOV \\ Naval Ocean-Atmospheric Prediction Laboratory, Department of Oceanography, Naval Postgraduate School, Monterey, California, \\ and Marine Hydrophysical Institute, Sevastopol, Ukraine \\ Oleg V. MelnichenKo \\ Marine Hydrophysical Institute, Sevastopol, Ukraine
}

(Manuscript received 9 October 2003, in final form 19 August 2004)

\begin{abstract}
Fall-winter recurrence of current reversal from westward to eastward is identified on the TexasLouisiana continental shelf using the current-meter [Texas-Louisiana Shelf Physical Oceanography Program (LATEX-A)] and near-surface drifting buoy [Surface Current and Lagrangian Drift Program (SCULP-1)] observations in 1993 and 1994. Reversal events roughly satisfy the Poisson distribution with one current reversal nearly every 12 days. Synoptic winds seem responsible for the current reversal events. Other processes such as offshore eddies shed from the Loop Current and river runoff are less important to change alongshore flow direction at synoptic scales. A statistical model is established to predict the synoptic current reversal using the surface wind observations.
\end{abstract}

\section{Introduction}

Bimodal cycles of geopotential anomaly and alternating cyclonic and anticyclonic regimes are important dynamical characteristics of the Texas-Louisiana continental shelf (TLCS) circulations (Boicourt et al. 1998). A cyclonic gyre usually dominates during fall and winter with a prevailing westward current on the TLCS (Cochrane and Kelly 1986). Satellite infrared images and Argos drifters in spring 1989 (Barron and Vastano 1994) and October 1992 (Vastano et al. 1995) show a coherent, 10-day surface current response over the entire TLCS to the establishment of a high atmospheric pressure system over the Gulf of Mexico. An alongshore westward current may stop with possibility of reversal into an eastward current when northerly winds are sustained over a few days (Nowlin et al. 1998) or when an anticyclonic circulation regime replaces the dominating cyclonic regime over the TLCS in summer (Oey 1995; Li et al. 1997; Cho et al. 1998; Ohlmann et al. 2001).

Corresponding author address: Dr. Peter C. Chu, Department of Oceanography, Naval Postgraduate School, Monterey, CA 93943. E-mail: chu@nps.navy.mil
Questions arise: Do the TLCS current reversals (from westward to eastward) occur on the synoptic timescale that dominates the surface wind forcing? If so, what are the characteristics of the TLCS current reversals? What is the major forcing to cause the synoptic TLCS current reversal? To answer these questions, establishment of temporally varying current velocity data with high spatial resolution is needed.

In this study, the optimal spectral decomposition (OSD) method (Chu et al. 2003a,b) is used to process the current velocity data collected from the TexasLouisiana Shelf Physical Oceanography Program (LATEX-A) and the first segment of the Surface Current and Lagrangian Drift Program (SCULP-1) into temporally-spatially gridded data. With this dataset, the TLCS current structure and reversal may be identified. In conjunction with the local surface wind data, the wind effect on the TLCS synoptic current reversal may also be identified.

\section{Data}

Data for the TLCS fall-winter current reconstruction were collected from 31 near-surface current-meter moorings during LATEX-A at $10-15-\mathrm{m}$ depths (Cho et 


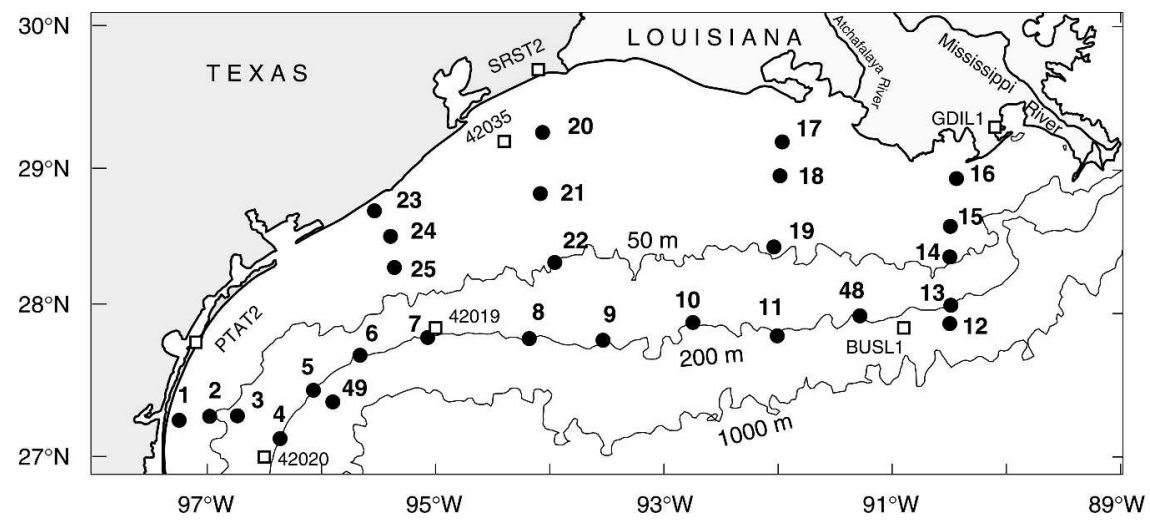

FIG. 1. Geography and topography of Texas-Louisiana continental shelf, LATEX-A current-meter stations (filled circles), and meteorological buoys (open squares).

al. 1998) from April 1992 to December 1994 (Fig. 1) and from drifting buoys deployed during SCULP-1 (Ohlmann et al. 2001) from October 1993 to July 1994. The current-meter moorings sample the current velocity (speed and direction), temperature, and salinity every $5 \mathrm{~min}$ to $2 \mathrm{~h}$ (mostly $30 \mathrm{~min}$ ). The drifting buoys record the location at various times with inhomogeneous area coverage. The data were interpolated into a uniform temporal grid with a time step of $3 \mathrm{~h}$.

Meteorological data are from seven buoys (represented by squares in Fig. 1) from the National Data Buoy Center (C-Man) in the LATEX-A area. Wang et al. (1998) have estimated the zero-crossing spatial scales of winds $(\sim 350 \mathrm{~km})$ and other meteorological parameters over the TLCS shelf after analyzing extensively the meteorological data collected during the LATEX-A Program observation period. Since the scale of the atmospheric systems is larger than the scale of the continental shelf, the horizontal mean wind speed and direction from seven buoys are used for analysis.

\section{OSD method}

A low-pass (40 h) filter is used to get rid of energetic shelf oscillations caused by tides, inertial currents, and sea breeze effects from the original current mooring and drifter observational data (Chen et al. 1996; DiMarco et al. 2000). The filtered data are reconstructed $\left(0.1^{\circ} \times 0.1^{\circ}\right.$ grid) using the OSD method (Chu et al. 2003a, b).

Between April and December 1992 the LATEX-A current-meter data have good temporal-spatial coverage, but the SCULP-1 drifter data are not available. To reduce the reconstruction error caused by spatial sparseness and temporal interruption in the observational data ( 8 times daily), three periods (238 days) are selected with reasonable reconstructed near-surface circulation: (i) from 21 January to 21 May 1993 (121 days, 968 observations), (ii) from 19 December 1993 to 17 February
1994 (61 days, 488 observations), and (iii) from 5 October to 29 November 1994 (56 days, 448 observations).

\section{OSD reconstruction skill}

Reconstruction of currents from sparse and noisy observational data is an ill-posed problem that may not have a unique solution. Theoretical and practical evaluations are conducted. The theoretical evaluation is to check if the solution is robust to sample size and noise level and asymptotically stable, that is, if the reconstructed solution homogeneously converges to an optimal solution when the number of observations tends to infinity (Chu et al. 2003a,b).

For evaluation, the LATEX-A data before the reconstruction are divided into testing and working data. The testing data are data collected at four currentmeter stations: 2, 4, 18, and 24 (Fig. 1). The working data are data collected at all the other current-meter stations. The working data are used for OSD reconstruction and the testing data are used for OSD verification.

Let $\left[u_{i}^{(\mathrm{rec})}, v_{i}^{(\mathrm{rec})}\right]$ and $\left[u_{i}^{(\mathrm{obs})}, v_{i}^{(\mathrm{obs})}\right]$ be $i$ th reconstructed and observed zonal and latitudinal velocity components (after a 40-h low-pass filter) at the testing stations. Figures 2-5 show $\left[u_{i}^{(\mathrm{rec})}, v_{i}^{(\mathrm{rec})}\right]$ (dashed curve) and $\left[u_{i}^{(\text {obs })}, v_{i}^{(\text {obs })}\right]$ (solid curve) at the four testing stations. The relative rms errors,

$$
\begin{aligned}
\operatorname{rrmse}(u) & =\frac{\sqrt{\sum_{i}\left[u_{i}^{(\mathrm{rec})}-u_{i}^{(\mathrm{obs})}\right]^{2}}}{\sqrt{\sum_{i}\left[u_{i}^{(\mathrm{obs})}\right]^{2}}} \text { and } \\
\operatorname{rrmse}(v) & =\frac{\sqrt{\sum_{i}\left[v_{i}^{(\mathrm{rec})}-v_{i}^{(\mathrm{obs})}\right]^{2}}}{\sqrt{\sum_{i}\left[v_{i}^{(\mathrm{obs})}\right]^{2}}},
\end{aligned}
$$

are $17.1 \%$ and $7.3 \%$ at station 2 , are $3.4 \%$ and $13 \%$ at station 24, and are larger at stations 4 and 18 (Table 1). 

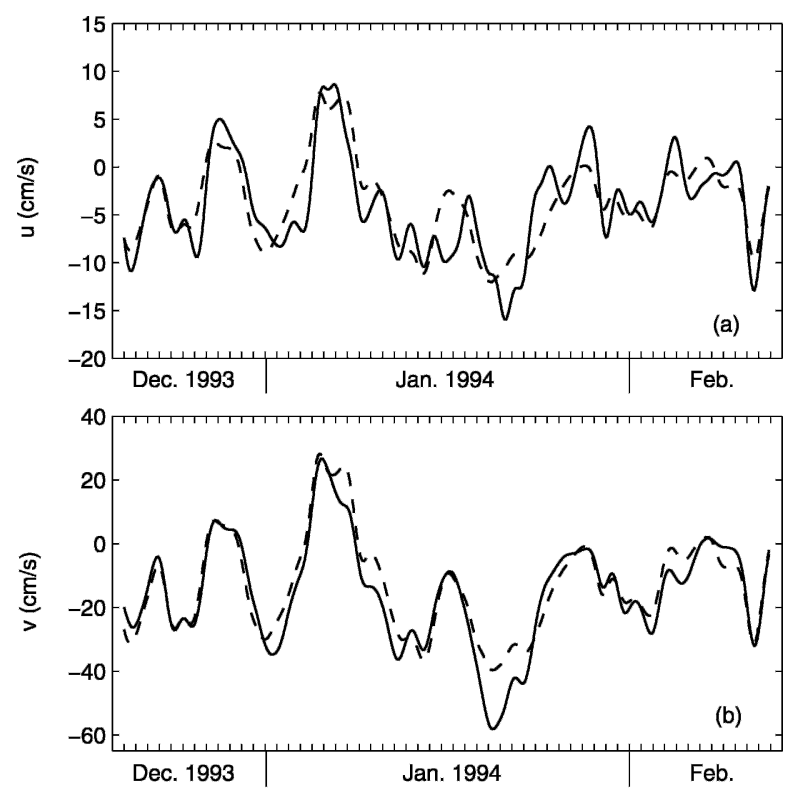

FIG. 2. OSD reconstructed (dashed line) and observed (black line, after 40-h low-pass filter) (a) zonal and (b) latitudinal velocity components at the stations not used for the reconstruction: station 2.

\section{Fall-winter synoptic current reversal}

Trajectories of 77 SCULP-I drifting buoys show current reversals during 2-7 January 1994 (Fig. 6). The OSD reconstructed horizontal velocity vector field
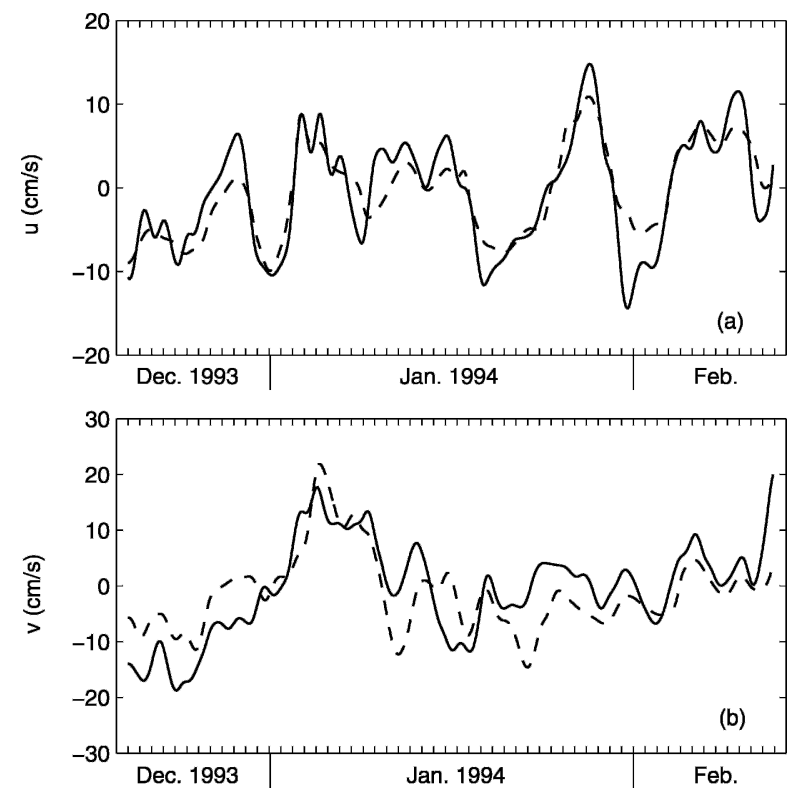

FIG. 3. OSD reconstructed (dashed line) and observed (black line, after 40-h low-pass filter) (a) zonal and (b) latitudinal velocity components at the stations not used for the reconstruction: station 4.
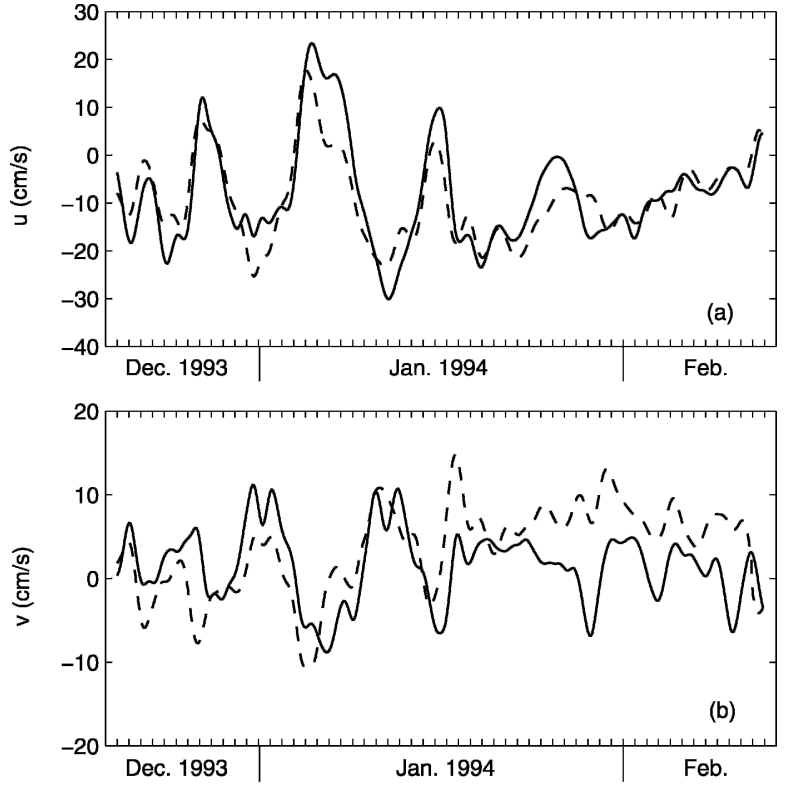

FIG. 4. OSD reconstructed (dashed line) and observed (black line, after 40-h low-pass filter) (a) zonal and (b) latitudinal velocity components at the stations not used for the reconstruction: station 18.

shows dynamical characteristics of the synoptic current. The flow pattern on 30 December 1993 is characterized as a cyclonic gyre with a strong westward TLCS flow as the northern flank (Fig. 7a). This current completely reverses toward the east on 3 January 1994 (total re-
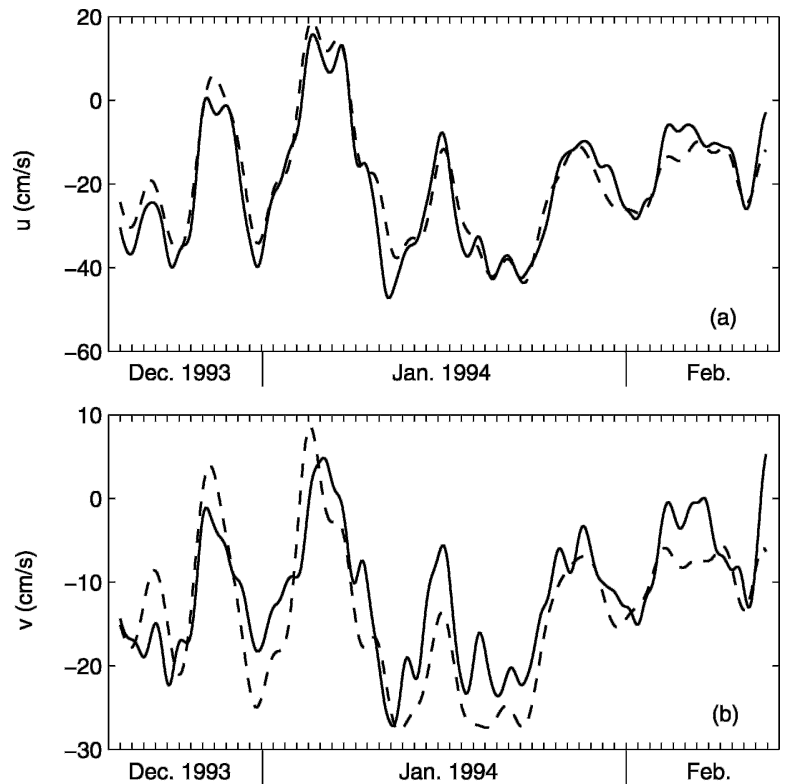

FIG. 5. OSD reconstructed (dashed line) and observed (black line, after 40-h low-pass filter) (a) zonal and (b) latitudinal velocity components at the stations not used for the reconstruction: station 24. 
TABLE 1. Relative rms error at the four testing stations.

\begin{tabular}{ccc}
\hline \hline & \multicolumn{2}{c}{ Rms error (\%) } \\
\cline { 2 - 3 } Mooring station & \multicolumn{1}{c}{$u$} \\
\hline 2 & 17.1 & 7.3 \\
4 & 14.9 & 21.4 \\
18 & 11.8 & 27.6 \\
24 & 3.4 & 13.0 \\
\hline
\end{tabular}

versal, Fig. 7b), and the cyclone reoccurs (6 January 1994) with the westward current at the north-central shelf and the eastward currents at the western shelf and shelf break (partial reversal, Fig. 7c). The total reversal has a width up to 200-m isobath (depth for shelf break), and the partial reversal has a width smaller than 200-m isobath. During that period (30 December 1993-6 January 1994) southwesterly winds prevail, and no evident offshore eddy appears at the shelf edge.

Recurrence of the TLCS current reversals is estimated using the OSD reconstructed velocity fields (238 days of duration). A period $T$ (from 5 to 40 days) is selected as the time window. The number of total (or partial) reversals is counted in each $T$ day window. Let $\left(n_{0}, n_{1}, n_{2}\right)$ be the numbers of 0 current reversal, 1 current reversal, and 2 current reversals, and $m$ be all realizations for a given $T$-day window. The probabilities for 0,1 , and 2 current reversals are calculated by

$$
P_{0}(T)=\frac{n_{0}}{m}, \quad P_{1}(T)=\frac{n_{1}}{m}, \quad \text { and } \quad P_{2}(T)=\frac{n_{2}}{m},
$$

where $P_{0}(T), P_{1}(T)$, and $P_{2}(T)$ depend on the period $T$ (Fig. 8). With $T$ larger than 20 days, the probability for zero current reversal is less than 0.2 . With $T$ of 15 days, the probability for one reversal reaches 0.5 . The probability $P_{k}(T)$ is fitted to the Poisson distribution (Fig. 8)

$$
P_{k}(T)=\frac{1}{k !}(\mu T)^{k} \exp (-\mu T), k=0,1,2,
$$

where $\mu=0.08$ day $^{-1}$ is the mean rate of current reversal. The probability of time interval between successive reversals $(\tau)$ is estimated using the Poisson distribution (2) (Tikhonov and Khimenko 1998),

$$
p(\tau)=\mu \exp (-\mu \tau),
$$

which shows that the synoptic reversal is not a rare event. Its characteristics can be obtained from analysis of temporal and spatial current variability.

\section{Empirical orthogonal functions}

The empirical orthogonal function (EOF) method (Preisendorfer 1988) is used to analyze the temporal and spatial variability of the three subdatasets of OSD reconstructed velocity. The first EOF (Table 2) is able to account for $80.2 \%$ variance for the first period (21 January-21 May 1993), 77.1\% for the second period (19 December 1993-12 February 1994), and 74.4\% for the third period (5 October-29 November 1994). Therefore, the velocity field can be roughly represented by

$$
\tilde{\mathbf{u}}(x, y, t)=\overline{\mathbf{u}}(x, y)+A_{1}(t) \mathbf{u}_{1}(x, y)
$$

for each period. Here, $\overline{\mathbf{u}}(x, y)$ is the mean velocity (background circulation) $\mathbf{u}_{1}(x, y)$ is the EOF-1 mode (nondimensional), and $A_{1}(t)$ is its principal component.

The background velocity field $\overline{\mathbf{u}}(x, y)$ presents a cyclonic circulation pattern (Fig. 9) with mean westward TLCS currents similar to Cho et al.'s (1998) results. During the first period, cyclogenesis over the shelf produced a strong winter storm (then called the "Storm of the Century") of 12-16 March 1993, which strongly affected currents during this month. The mean pattern

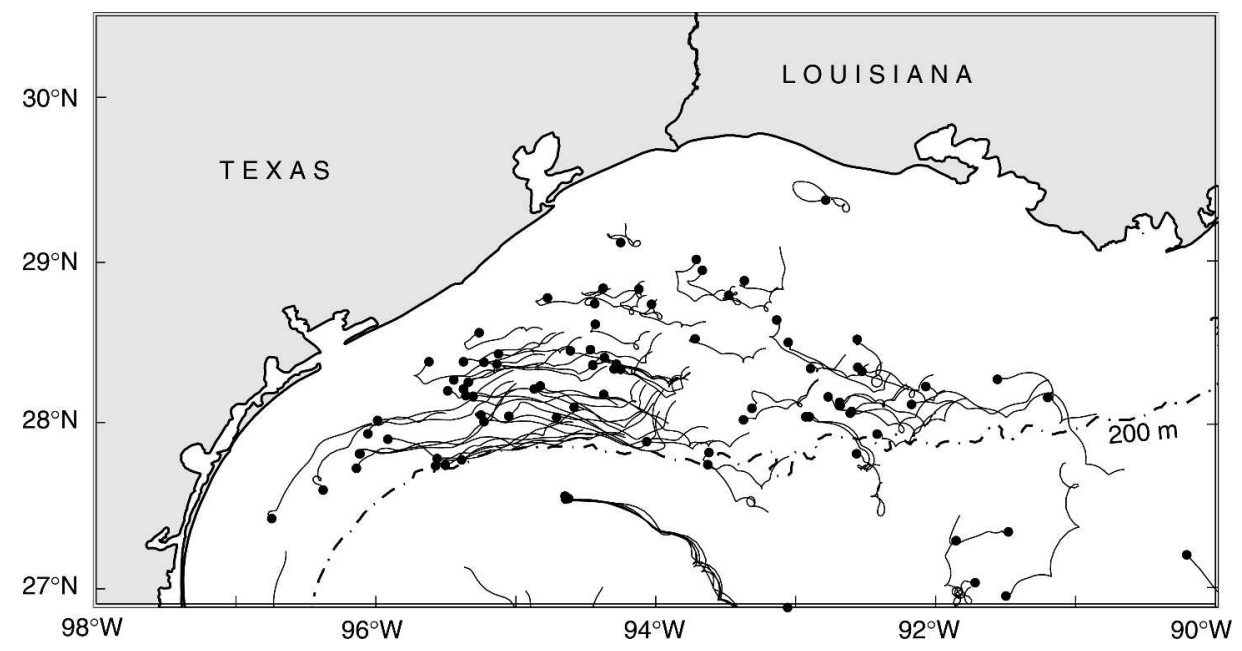

FIG. 6. TLCS current reversals detected from SCULP-1 buoy trajectories during 2-7 Jan 1994. The black dots show the starting positions of buoys. 

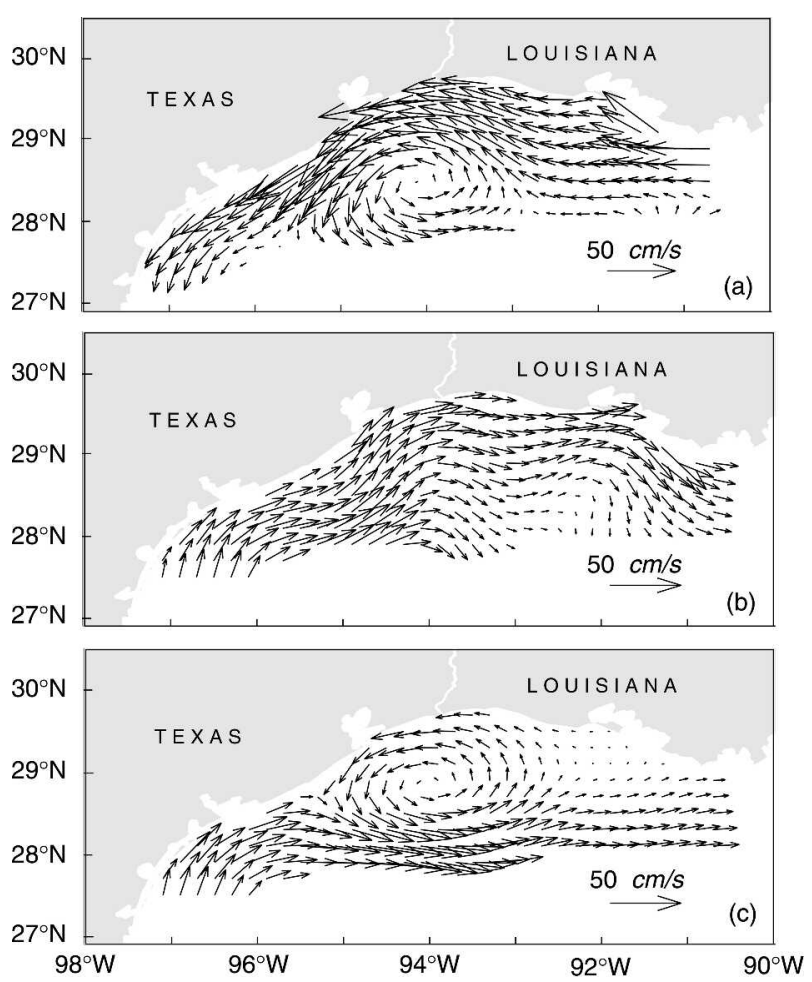

FIG. 7. OSD reconstructed circulation: (a) cyclonic gyre on 30 Dec 1993, (b) total reversal on 3 Jan 1994, and (c) partial reversal on 6 Jan 1994.

shown in Fig. 9a strongly resembles the cyclonic atmospheric pattern during the time of the storm.

The EOF-1 mode presents an anticyclonic circulation pattern for positive $A_{1}(t)$ (Fig. 10) with mean eastward TLCS currents. Comparison is conducted between the reconstructed fields from two independent observational datasets collected during the same period. High coherence (correlation coefficient $\sim 0.92$ ) is found in $A_{1}(t)$ from 19 December 1993 to 12 February 1994 between using the LATEX-A current-meter mooring data (solid curve) and the SCULP-I drifter (dashed curve) data (Fig. 11). This shows the reconstruction skill of the OSD method.

The TLCS current reversal depends on the relative magnitudes of $\overline{\mathbf{u}}(x, y)$ and $A_{1}(t) \mathbf{u}_{1}(x, y)$ [positive $A_{1}(t)$ ]. Since $\overline{\mathbf{u}}(x, y)$ and $\mathbf{u}_{1}(x, y)$ are totally opposite on the TLCS, total and partial current reversals can be simply represented by two thresholds $S_{t}$ and $S_{p}$. The background current velocity field $\overline{\mathbf{u}}(x, y)$ changes from one period to the other (Fig. 9), the thresholds for the reversal also vary. For the first period (21 January-21 May 1993), the two thresholds are given by

$$
S_{t}=23 \text { and } S_{p}=8\left(\mathrm{~m} \mathrm{~s}^{-1}\right) .
$$

Eight total reversal events are identified during the first period (Fig. 12a): 7-9 and 12-13 February, 5-11 and 14-16 March, 31 March-3 April, 9-12 and 14-17 April, and 9-12 May 1993.
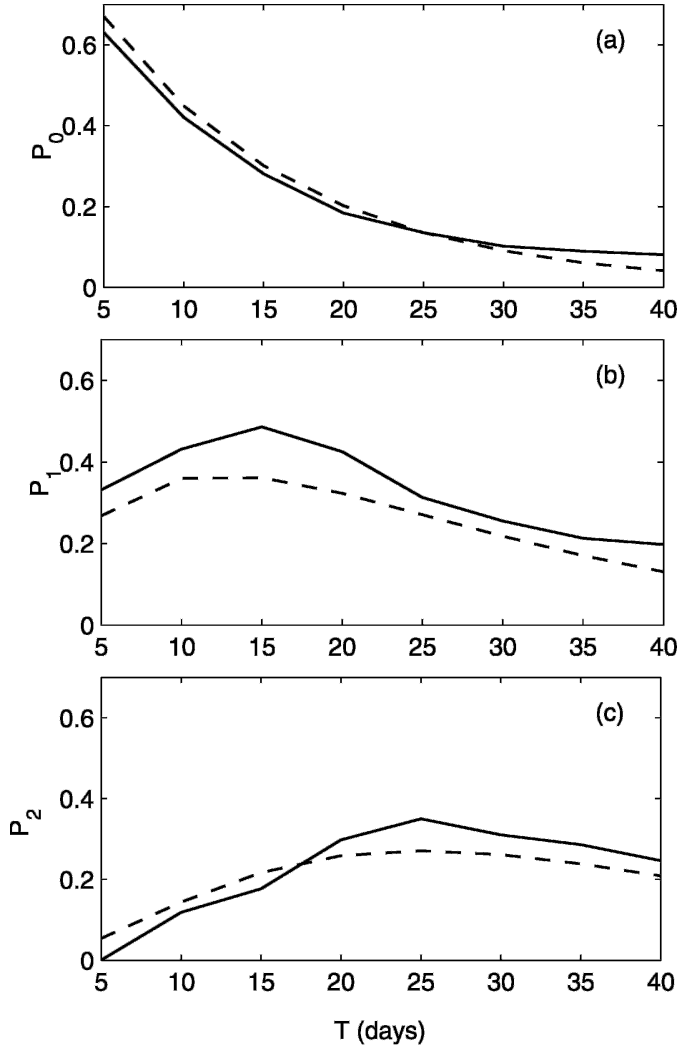

FIG. 8. Empirically (dashed curve) and theoretically (Poisson distribution, solid curve) estimated recurrence probabilities: (a) $P_{0}(T)$, (b) $P_{1}(T)$, and (c) $P_{2}(T)$ as functions of duration $T$.

Temporally varying the weight ratio between the square of principal component PC-1 to the sum of the squares of PC-2 to PC-6, (Fig. 12b),

$$
\eta(t)=\left[A_{1}(t)\right]^{2} / \sum_{n=2}^{6}\left[A_{n}(t)\right]^{2}
$$

represents importance of the EOF-1 mode relative to higher EOF modes. The total and partial current reversal events correspond to large $\eta$ values.

\section{Wind-driven mechanism}

Wind, freshwater influx, and offshore eddies affect TLCS circulations (Boicourt et al. 1998). What is the

TABLE 2. Variance of the first six EOFs.

\begin{tabular}{cccc}
\hline \hline & \multicolumn{3}{c}{ Variance (\%) } \\
\cline { 2 - 4 } EOF & 21 Jan 1993- & 19 Dec 1993- & 5 Oct- \\
\hline 1 & 21 May 1993 & 17 Apr 1994 & 29 Nov 1994 \\
2 & 80.2 & 77.1 & 74.4 \\
3 & 10.1 & 9.5 & 9.3 \\
4 & 3.9 & 5.6 & 6.9 \\
5 & 1.4 & 3.3 & 4.6 \\
6 & 1.1 & 1.4 & 2.3 \\
\hline
\end{tabular}



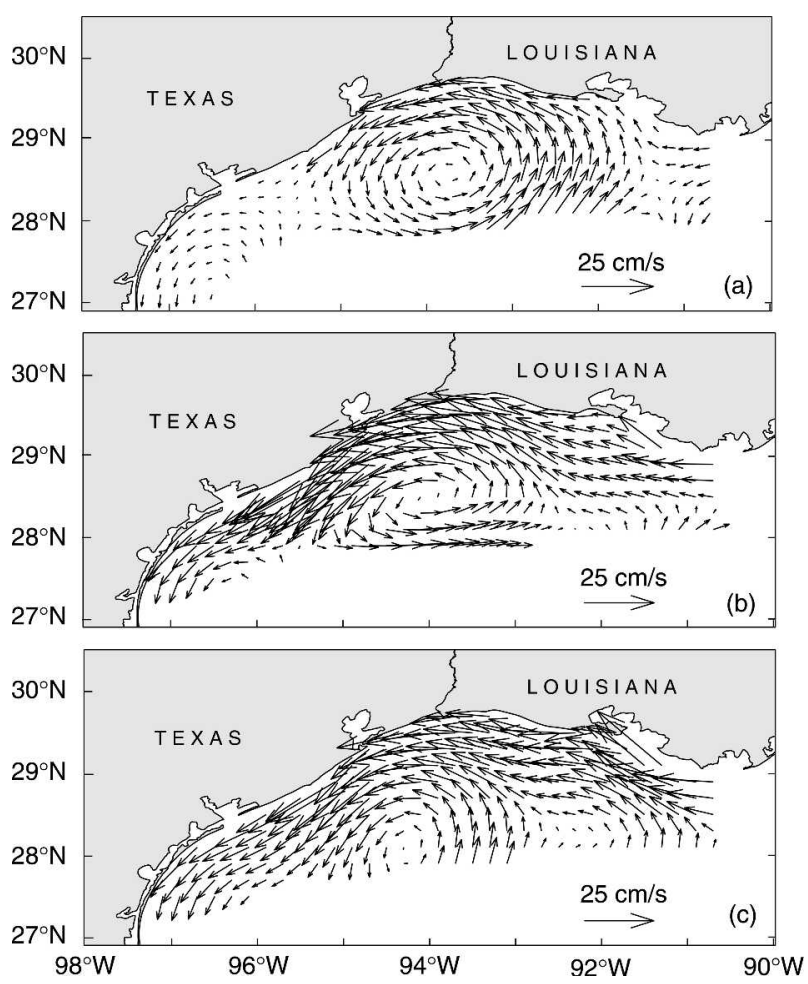

FIG. 9. OSD reconstructed mean circulation $\overline{\mathbf{u}}(x, y)$ during (a) first period (21 Jan-21 May 1993), (b) second period (19 Dec 1993-12 Feb 1994), and (c) third period (5 Oct-29 Nov 1994).

major forcing for the TLCS current reversal? Analysis of reversal events for the first period (January 1993May 1993) is taken as a sample for the study. The horizontally averaged surface zonal and latitudinal wind components $[U(t), V(t)](8$ times daily) are calculated from the seven meteorological buoys. The TexasLouisiana coastline is slanted roughly $17^{\circ}$ to the zonal direction. The alongshore wind $\left[U_{\text {als }}(t)\right]$ is the projection of the surface wind vector to the axis rotated $17^{\circ}$ anticlockwise from the latitude. Comparing $U_{\text {als }}(t)$ with $A_{1}(t)$ (Figs. 12a,c), the total reversals on TLCS are associated with eastward alongshore winds at wind speeds greater than $2 \mathrm{~m} \mathrm{~s}^{-1}$. The correlation coefficient between $U_{\text {als }}(\mathrm{t})$ and $A_{1}(t)$ is 0.82 for the first period (sample size: 968), 0.83 for the second period (sample size: 488 ), and 0.74 for the third period (sample size: 448). For significance level $\alpha=0.005$, the critical value of the sample correlation coefficient is 0.254 for sample size of 102 and is less than 0.254 for sample size larger than 102 .

Connection between $U_{\text {als }}(t)$ and $A_{1}(t)$ (sampling rate of 8 times per day) is further investigated using their temporally varying spectra (Fig. 13) from the Morlet wavelet transform (Morlet et al. 1982),

$$
\Phi(t)=\pi^{-4} \exp \left(i m t-t^{2} / 2\right), \quad m=6,
$$

which is similar at frequencies between 0.2 and 0.05 cpd. This may confirm the connection between synoptic current reversals and alongshore surface winds.
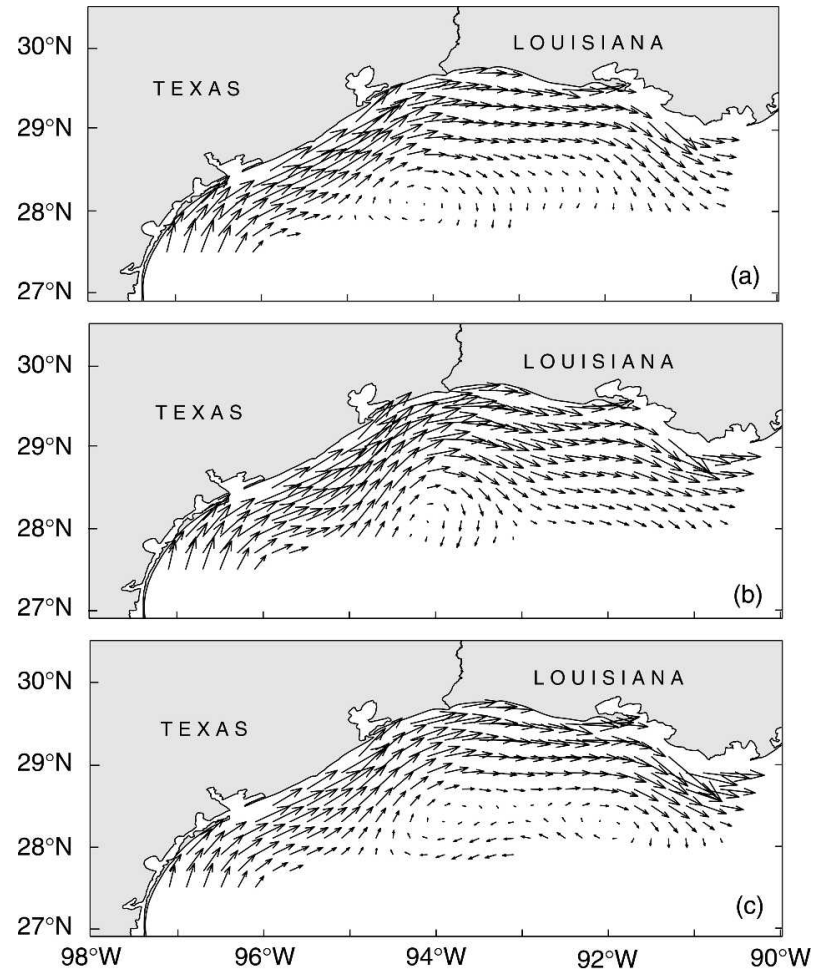

FIG. 10. OSD reconstructed first EOF mode $\mathbf{u}_{1}(x, y)$ during (a) first period (21 Jan-21 May 1993), (b) second period (19 Dec 1993-12 Feb 1994), and (c) third period (5 Oct-29 Nov 1994).

\section{Statistical prediction model}

For predicting synoptic current reversals, the observed surface wind $[U(t), V(t)]$ (not alongshore and cross-shore components) is used with a simple statistical model,

$$
A_{1}(t)=\alpha[U(t)-\bar{U}]+\beta[V(t)-\bar{V}]+\gamma,
$$

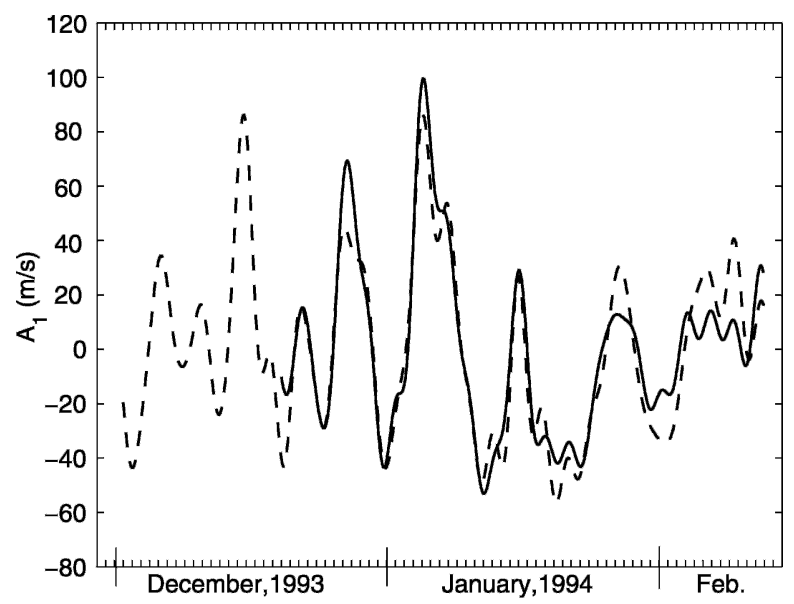

FIG. 11. OSD reconstructed $A_{1}(t)$ using the LATEX-A currentmeter mooring (solid curve) and the SCULP-I drifter (dashed curve) data. 

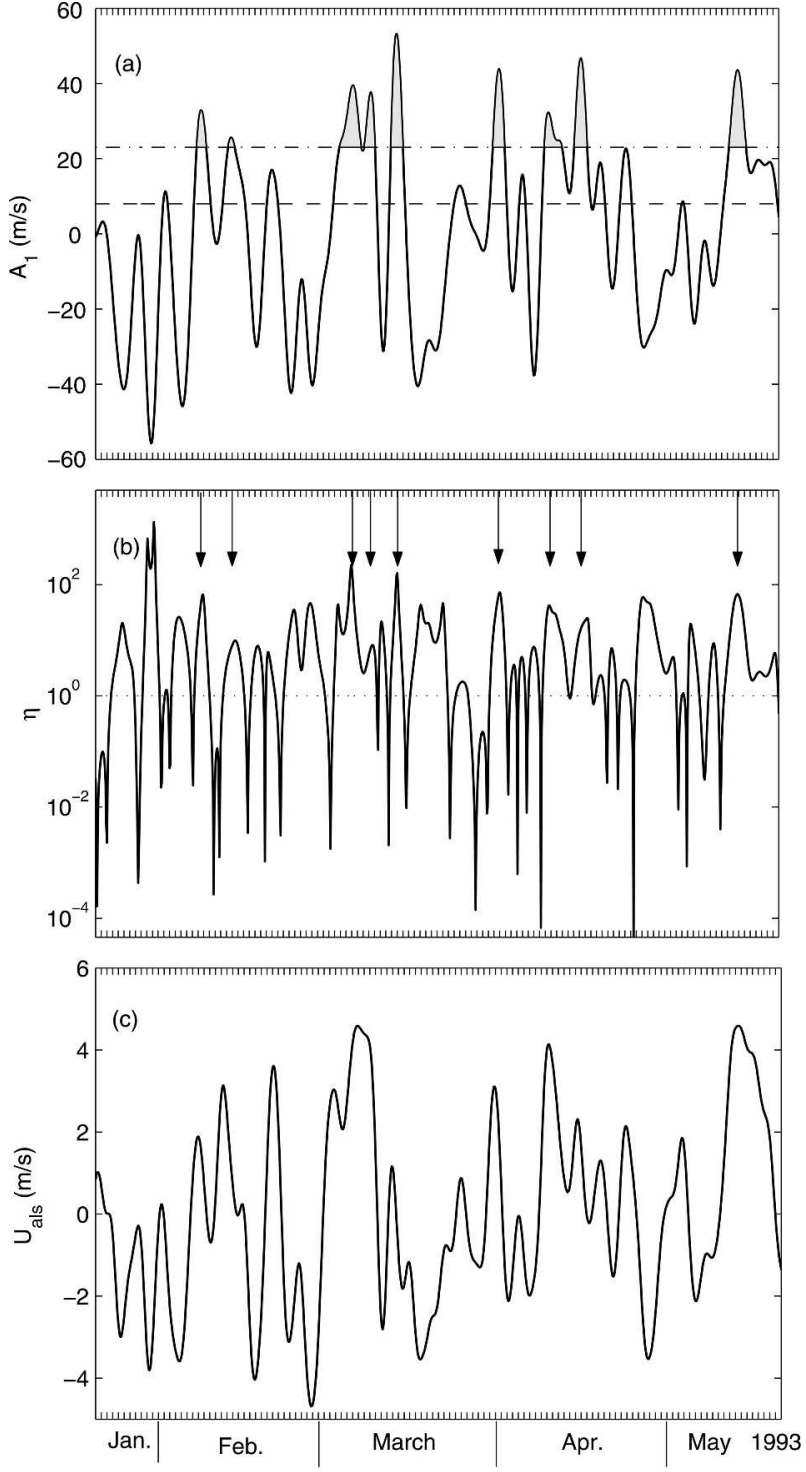

FIG. 12. Time series of (a) $A_{1}(t)\left(\mathrm{m} \mathrm{s}^{-1}\right)$, (b) $\eta(t)$, and (c) $U_{\text {als }}(t)$ $\left(\mathrm{m} \mathrm{s}^{-1}\right)$. Eight occurrences of high $A_{1}$ values (arrows), which represent the TLCS total current reversals, correspond to high values of $\eta$ and $U_{\text {als. }}$. Here, dash-dotted and dashed lines are the thresholds for total and partial current reversals, respectively.

where $\bar{U}$ and $\bar{V}$ are temporally averaged wind speed and $\alpha, \beta$, and $\gamma$ are regression coefficients. The regression coefficients are calculated from the data collected during the second period (19 December 1993-17 February 1994):

$$
\alpha=9.032, \quad \beta=3.754, \quad \text { and } \quad \gamma=0.209 \mathrm{~m} \mathrm{~s}^{-1} \text {. }
$$

Nonzero regression coefficient $(\gamma \neq 0)$ suggests that the wind forcing may not be the only factor causing the synoptic TLCS current reversal. This is because the current reversal may occur $\left(A_{1} \neq 0\right)$ even if there is no
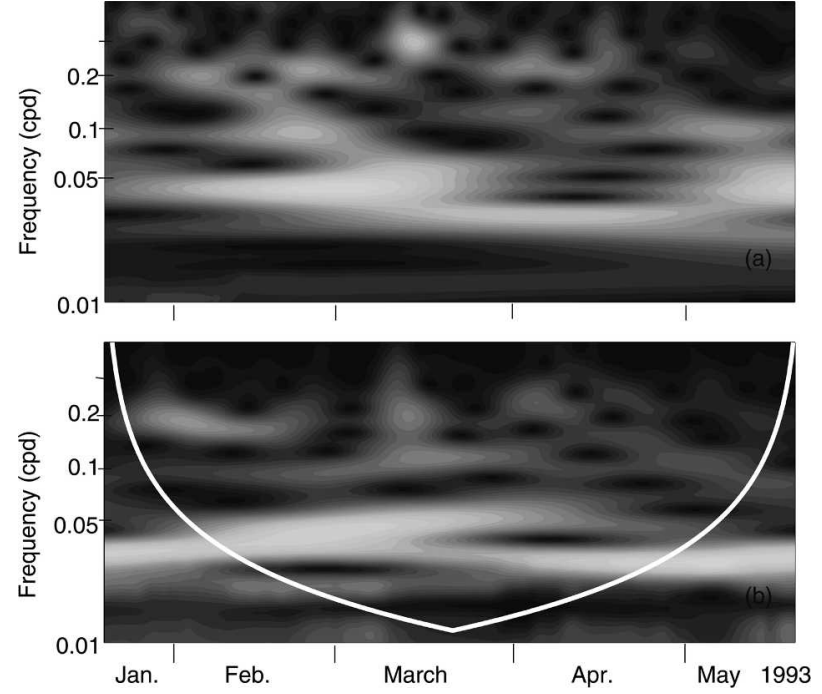

FIG. 13. Morlet wavelet spectra of (a) $U_{\text {als }}(t)$ and (b) $A_{1}(t)$ calculated using the data collected during the first period (21 Jan-21 May 1993). The cone of influence for $A_{1}(t)$ is denoted by the white curve above which the edge effect (due to zero-padding ends) is greatly reduced.

wind anomaly $[U(t)=\bar{U}, V(t)=\bar{V}]$. Since the two regression coefficients for the wind speeds are not small $(\alpha=9.032, \beta=3.754)$, contribution of $\gamma\left(\sim 0.2 \mathrm{~m} \mathrm{~s}^{-1}\right)$ to $A_{1}(t)$ is negligible when the surface winds are stronger than $0.4 \mathrm{~m} \mathrm{~s}^{-1}$.

The Mississippi-Atchafalaya River discharge increases the cross-shelf salinity gradients. With salinity increasing offshore, the cyclonic circulation enhances (Chen et al. 2000) and stronger eastward (alongshore) winds are required to reverse the mean westward TLCS; that is, the threshold of reversal is higher.

The offshore eddies of the Loop Current origin may also affect the synoptic current reversal. To investigate such an effect, the SCULP-I drifter data are reconstructed for weak alongshore winds $\left(U_{\text {alg }}\right.$ around 0.4 $\mathrm{m} \mathrm{s}^{-1}$; Fig. 14) and strong alongshore winds $\left(U_{\mathrm{alg}}>3.5\right.$ $\mathrm{m} \mathrm{s}^{-1}$; Fig. 15). For weak alongshore winds, offshore eddies drive strong fluctuations of the alongshore flow across the shelf and interact with the alongshore jet (Fig. 14b). For strong alongshore winds, the offshore eddies appear. They can only cause a local acceleration or deceleration of alongshore flow but cannot change its direction [small value of $\gamma$ in (7)].

For strong alongshore winds, offshore eddies do not appear on the sea surface (Fig. 15a) but occur on the upper slope as counterrotating pairs. Such offshore eddies may deflect the eastward currents into southeastward currents in the western TLCS (Fig. 15b).

The statistical prediction model (7)-(8) was established using the data collected during the second period (19 December 1993-17 February 1994). The data collected during the third period (5 October-29 November 1994) are taken as the independent dataset to verify the 

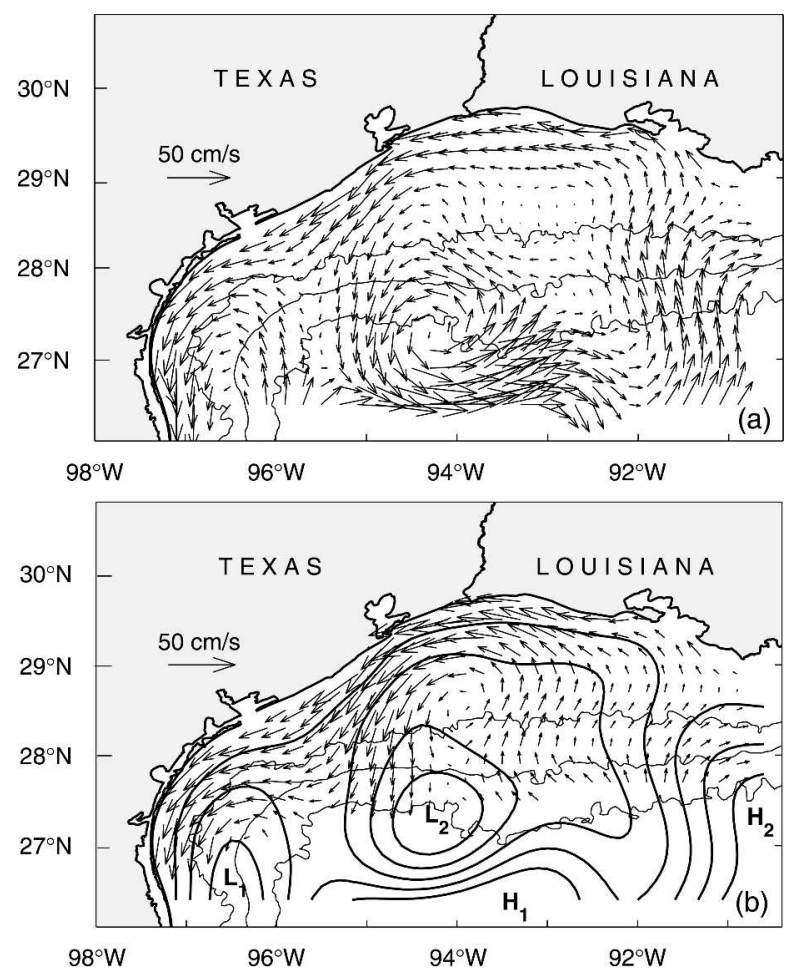

FIG. 14. OSD reconstructed circulation with northeasterly winds (around $0.4 \mathrm{~m} \mathrm{~s}^{-1}$ ) at (a) surface and (b) 15-m depth from SCALP-I and LATEX-A observational data at 1200 UTC $10 \mathrm{Feb}$ 1994. Streamfunction at $15-\mathrm{m}$ depth (thick curves) shows multieddy structure with two cyclonic eddies on the TLCS. The thin solid curves are isobaths at 50,200 , and $1000 \mathrm{~m}$.

statistical model. Figure 16 shows the comparison between the reconstructed (from the third period data, solid curve) and predicted (dashed curve) principal component, $A_{1}(t)$. The correlation coefficient between the two is 0.7 . The statistical model (7) predicted the occurrence of three total reversal events-14 October 1994, 23 October 1994, and November 1994-and the three partial reversal events-2, 7, and 23 November 1994. It failed to predict two partial reversal events: 4 and 10 November 1994.

\section{Conclusions}

The OSD method is used to analyze fall-winter LATEX-A and SCULP-1 data. Theoretical and practical evaluations show a high reconstruction skill of the OSD method. The mean OSD reconstructed horizontal velocity field shows a cyclonic circulation pattern near the Texas-Louisiana coast with the westward-flowing TLCS currents.

Relative rms errors between reconstructed and observed (not used for the reconstruction) zonal and latitudinal current velocity data are used to measure the OSD reconstruction skill. At station 24, the relative rms error is $3.4 \%$ for the zonal current and $13 \%$ for the
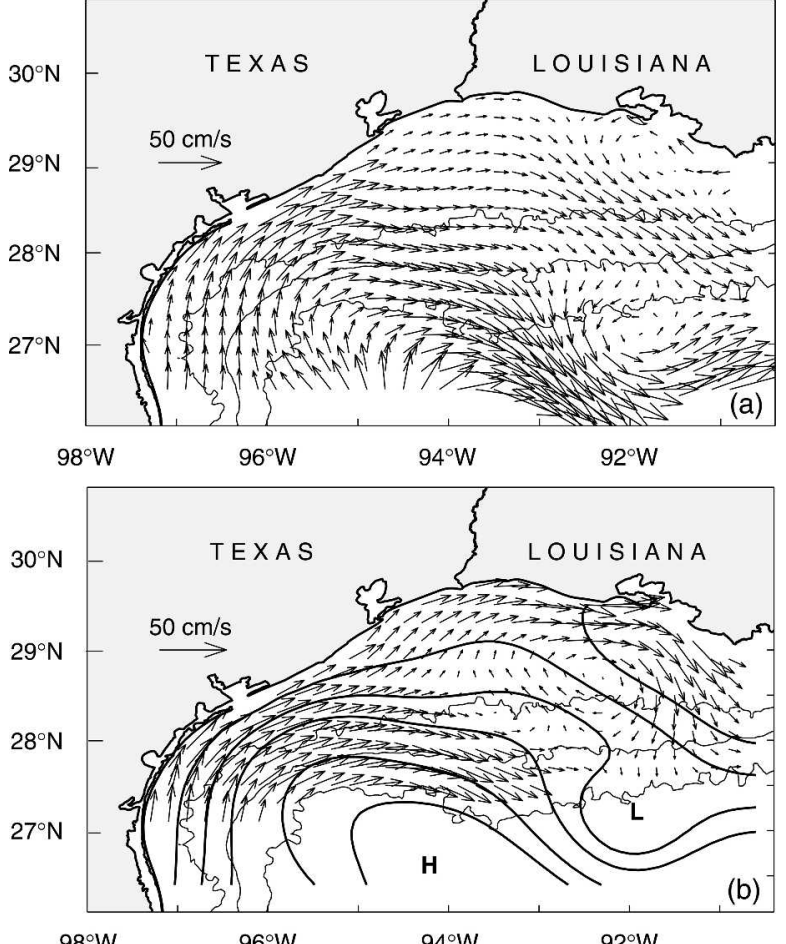

FIG. 15. OSD reconstructed circulation with southwesterly winds (above $3.5 \mathrm{~m} \mathrm{~s}^{-1}$ ) at (a) surface and (b) $15-\mathrm{m}$ depth from SCALP-I and LATEX-A observational data at 1800 UTC 4 Jan 1994. Streamfunction at $15-\mathrm{m}$ depth (thick curves) shows an anticyclonic eddy on the TLCS. The thin solid curves are isobaths at 50, 200, and $1000 \mathrm{~m}$.

latitudinal current. The reconstructed velocity fields are consistent using the LATEX-A current-meter mooring data and the SCULP-I drifter data collected at the same period with high-coherence (correlation coefficient

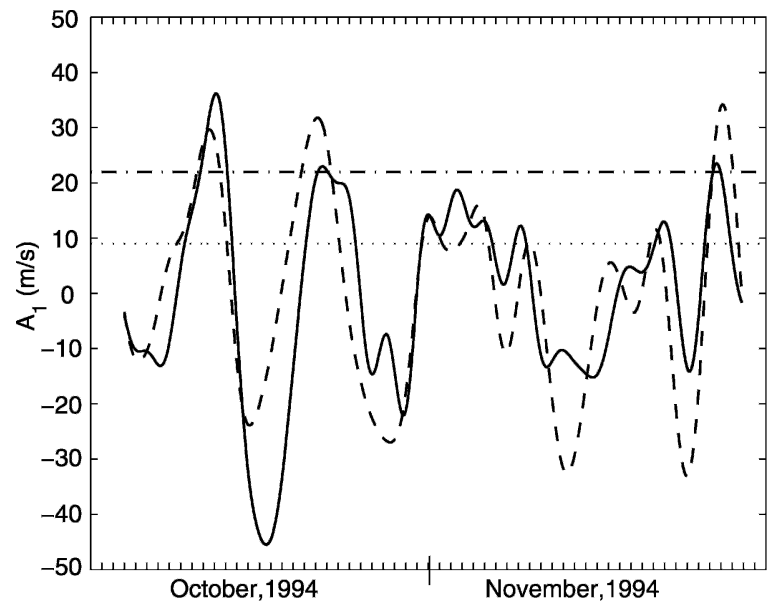

FIG. 16. Predicted (dashed curve) and OSD reconstructed (solid curve) $A_{1}(t)\left(\mathrm{m} \mathrm{s}^{-1}\right)$ during Oct-Nov 1994. Dash-dotted and dotted lines indicate thresholds for the total and partial TLCS current reversals. 
0.92) $A_{1}(t)$ from 19 December 1993 to 12 February 1994. This also shows a high reconstruction skill of the OSD method.

Temporally varying OSD reconstructed velocity fields show that the synoptic TLCS current reversal is not a rare event. Probability for its recurrence satisfies the Poisson distribution. With a period longer than 20 days, the probability for zero current reversal is less than 0.2 . With a period of 15 days, the probability for one reversal reaches 0.5 .

The EOF-1 mode (anticyclonic circulation pattern) accounts for $80.2 \%$ variance for the first period (21 January-21 May 1993), 77.1\% for the second period (19 December 1993-12 February 1994), and 74.4\% for the third period (5 October-29 November 1994). Occurrence of fall-winter total and partial synoptic current reversals on the TLCS (i.e., altering the mean cyclonic circulation pattern) depends on the value of the principal component corresponding to EOF-1. When the value exceeds a threshold, the synoptic current reversal occurs on TLCS. Eight total reversal events are all identified during the first period by this method: 7-9 and 12-13 February, 5-11 and 14-16 March, 31 March-3 April, 9-12 and 14-17 April, and 9-12 May 1993.

Alongshore wind forcing is the major factor causing the synoptic current reversal. Other factors, such as the Mississippi-Atchafalaya River discharge and offshore eddies of Loop Current origin, may affect the reversal threshold but cannot cause the synoptic current reversal.

A simple statistical model is developed to predict the total and partial reversals of the TLCS currents using the surface winds. The model evaluation using independent dataset shows high capability to predict the total synoptic reversals, but low capability to predict the partial reversals.

The Texas-Louisiana shelf is strongly bimodal, having a summer and nonsummer seasonal oscillation. For the summer season, the predominant current direction is eastward, opposite to the winter season. Therefore, only the fall-winter data are analyzed in this study. A similar analysis may be used to see if synoptic TLCS current reversal occurs in summer.

Acknowledgments. This work was supported by the Office of Naval Research, Naval Oceanographic Office, and the Naval Postgraduate School. Authors L. Ivanov and O. Melnichenko thank the Civil Research Development Foundation for supporting this work through Award UG2-2079. We thank Dr. Lynne Talley and two anonymous reviewers for their invaluable comments.

\section{REFERENCES}

Barron, C. N., and A. C. Vastano, 1994: Satellite observations of surface circulation in the northwestern Gulf of Mexico during March and April 1989. Cont. Shelf Res., 14, 607-628.

Boicourt, W. C., W. J. Wiseman Jr., A. Valle-Levinson, and L. Atkinson, 1998: Continental shelf of the southeastern United States and the Gulf of Mexico: In the shadow of the western boundary current. The Sea, A. R. Robinson and K. H. Brink, Eds., Regional Studies and Syntheses, Vol. 11, John Wiley and Sons, 135-175.

Chen, C., R. O. Reid, and W. D. Nowlin Jr., 1996: Near-inertial oscillations over the Texas-Louisiana shelf. J. Geophys. Res., 101, 3509-3524.

Chen, X., S. E. Lohrenz, and D. A. Wiesenberg, 2000: Distribution and controlling mechanisms of primary production on the Louisiana-Texas continental shelf. J. Mar. Syst., 25, 179207.

Cho, K., R. O. Reid, and W. D. Nowlin, 1998: Objectively mapped stream function fields on the Texas-Louisiana shelf based on 32 months of moored current meter data. J. Geophys. Res., 103, $10377-10390$

Chu, P. C., L. M. Ivanov, T. M. Margolina, T. P. Korzhova, and O. V. Melnichenko, 2003a: Analysis of sparse and noisy ocean current data using flow decomposition. Part I: Theory. J. Atmos. Oceanic Technol., 20, 478-491.

$\longrightarrow,-$, and $\longrightarrow$, 2003b: Analysis of sparse and noisy ocean current data using flow decomposition. Part II: Applications to Eulerian and Lagrangian data. J. Atmos. Oceanic Technol., 20, 492-512.

Cochrane, J. D., and F. J. Kelly, 1986: Low-frequency circulation on the Texas-Louisiana shelf. J. Geophys. Res., 91, 10 64510659.

DiMarco, S. F., M. K. Howard, and R. O. Reid, 2000: Seasonal variation of wind-driven diurnal current cycling on the Texas-Louisiana continental shelf. Geophys. Res. Lett., 27, 1017 1020.

Li, Y., W. D. Nowlin Jr., and O. R. Reid, 1997: Mean hydrographic fields and their inter-annual variability over the Texas-Louisiana continental shelf in spring, summer, and fall. $J$. Geophys. Res., 102, 1027-1049.

Morlet, J., G. Arens, E. Fourgeau, and D. Giard, 1982: Wave propagation and sampling theory, Part I: Complex signal and scattering in multilayered media. Geophysics, 47 (2), 203-221.

Nowlin, W. D., Jr., A. E. Jochens, R. O. Reid, and S. F. DiMarco, 1998: Texas-Louisiana shelf circulation and transport processes study. Synthesis Report, Vol. 1, Texas A\&M University, $1-5$.

Oey, L., 1995: Eddy- and wind-forced shelf circulation. J. Geophys. Res., 100, 8621-8637.

Ohlmann, J. C., P. P. Niiler, C. A. Fox, and R. R. Leben, 2001: Eddy energy and shelf interactions in the Gulf of Mexico. $J$. Geophys. Res., 106, 2605-2620.

Preisendorfer, R. W., 1988: Principal Component Analysis in Meteorology and Oceanography, Elsevier, $425 \mathrm{pp}$.

Tikhonov, V. I., and V. I. Khimenko, 1998: Level-crossing problems for random process. Radiotekhnika I Elektronika, 43, 501-523.

Vastano, A. C., C. N. Barron Jr., and E. W. Shaar Jr., 1995: Satellite observations of the Texas Current. Cont. Shelf Res, 15, 729-754.

Wang, W., W. D. Nowlin Jr., and R. O. Reid, 1998: Analyzed surface meteorological fields over the northwestern Gulf of Mexico for 1992-94-Mean, seasonal, and monthly patterns. Mon. Wea. Rev., 126, 2864-2883. 